\title{
APROXIMACIONES TEÓRICAS Y EMPÍRICAS a la Relación DE Causalidad ENTRE DESIGUALDAD Y CRECIMIENTO: Un ANÁlisis para Colombia. 1985-2006
}

\author{
Por: Camilo Rey Sabogal
}

Artículo Recibido: Mayo 10 de 2008

Artículo Aprobado: Junio 13 de 2008

\section{RESUMEN}

Este trabajo pretende examinar las posibles relaciones de causalidad entre desigualdady crecimiento. Se describen los hallazgos de Simón Kuznets que le permitieron formular su Hipótesis de la curva U-Invertida, así como los principales enfoques teóricos y empíricos que se generaron a partir de dicha Hipótesis, a nivel internacional y nacional. Luego al analizar las cifras del país se encontró que el mecanismo de crecimiento actual se fundamenta en la promoción de la desigualdad. Finalmente, un análisis econométrico de las posibles relaciones, permitió extraer los siguientes hallazgos: a) El alto desempleo y la caída de los salarios reales determina la alta inequidad, b) La hipótesis de Kuznets se queda corta ante los nuevos hechos que han elevado nuevamente la desigualdad, y c) Existe evidencia de que la relación de causalidad va desde distribución hacia crecimiento y no al revés.

Palabras Claves: Desigualdad, crecimiento económico, Kuznets.

\section{ABSTRACT}

This paper aims to explore the possible causal relationship between inequality and growth. It describes the findings of Simon Kuznets that allowed him to state his inverted U-shaped curve hypothesis, as well as the major theoretical and empirical approaches that were generated from that hypothesis, internationally and nationally. After analyzing the country statistics, it was found that the current growth mechanism is based on the promotion of the inequality. Finally, an econometrics analysis of the possible relationships, allowed extracting the following findings: a) The high unemployment and falling real wages determines the high inequality. b) The Kuznets hypothesis is not enough regarding the new facts that have risen again inequality, 
and c) There is evidence that the causality runs from distribution to growth and not vice versa.

\section{Key Words: Inequality, economic growth, Kuznets}

\section{Introducción}

n los años recientes Colombia rompe dos records económicos importantes.
Por un lado presenta las tasas de crecimiento más elevadas de las últimas
tres décadas y a su vez registra los peores indicadores de desigualdad de los últimos 40 años. Aunque el hecho sorprende, tiene una explicación dentro del marco teórico de la política económica de los países pro Consenso de Washington, que supone la existencia de un trade-off entre desigualdad y crecimiento. Este discurso argumenta, que en un principio las capas bajas de la sociedad deben sacrificarse en aras de lograr la riqueza nacional, la cual posteriormente será derramada como una cascada desde las capas altas hacia abajo. Esta "ley" se fundamenta en dos planteamientos. Primero la existencia de otro famoso tradeoff entre eficiencia y equidad, el cual indica que, en búsqueda de igualdad, la transferencia de renta de los más ricos a los pobres reduciría la eficiencia, porque disminuye los incentivos para producir en los primeros y genera motivos para no trabajar en los segundos. El otro planteamiento es el hallazgo de Kuznets sobre una relación directa entre desigualdad y crecimiento en las primeras etapas del desarrollo y una relación inversa en etapas posteriores.

Sin embargo, las nuevas evidencias y las transformaciones económicas recientes exigen una revisión de la Hipótesis de Kuznets. En los años 70 el país entendía que ya estaba recibiendo los frutos de sus sacrificios en años anteriores, por lo cual el tema de la desigualdad pasó a un segundo plano en la agenda social colombiana (Londoño, 1995). Pero a finales de los 80 la historia dio otro giro. Se inició un proceso de apertura de mercados y la inequidad volvió a crecer. Nuevamente se exigió un sacrificio. La promesa: las mieles de la globalización no tardarán en derramarse a todas las capas de la sociedad.

En este trabajo, se pretende abordar los argumentos teóricos que fundamentan las relaciones de causalidad entre desigualdad y crecimiento, para luego efectuar ejercicios empíricos que arrojen pistas sobre la dirección de dicha causalidad en Colombia, y sobre el efecto de los nuevos acontecimientos de liberalización en el cumplimiento de la Hipótesis de Kuznets. 


\section{Contexto Teórico}

El análisis de las relaciones de causalidad o no causalidad entre desigualdad y crecimiento económico se pueden percibir desde la economía clásica, aunque aún de forma precaria. David Ricardo indica la importancia que tiene la distribución de la renta determinada a partir de la productividad de los factores sobre la acumulación de capital, como estrategia para el incremento de la producción. En Karl Marx se puede evidenciar que la desigualdad se genera por la propia dinámica del capitalismo, que exige mayor acumulación por parte de la clase capitalista a costa de la plusvalía extraída al trabajo del proletariado. Sin embargo, Marx supone que la evolución de las fuerzas productivas conducirá inevitablemente a una posterior redistribución de la propiedad de los medios de producción.

Por el contrario John M. Keynes resalta la baja propensión marginal a consumir de los ricos en comparación con la de los pobres, lo cual permite que los primeros destinen cada vez más al ahorro en perjuicio de la demanda agregada, mientras que los segundos por su condición de pobreza no logran incrementarla, dada su urgencia solo por bienes básicos. Por ello la redistribución de la renta a través de mejores salarios reales o de transferencias estatales permiten el aumento del consumo en la gran mayoría y por tanto el incentivo a la inversión para aumentar la producción.

Sin embargo, solo en la etapa de la segunda postguerra, en épocas de reconstrucción y recuperación, de forma consecuente con los acontecimientos del momento, la ciencia económica centra su atención en las causas del crecimiento económico de largo plazo y su caracterización, con el fin de encontrar factores que permitan acelerar su llegada en la mayoría de los países semidestruidos.

En este contexto Arthur Lewis (1954) evaluando un grupo de países pobres detectó que el alto desempleo permite que los sectores más productivos absorban mano de obra barata proveniente de los sectores tradicionales de baja productividad, pero que caracteriza este tipo de países. Durante el transcurso que hace la sociedad desde sectores con exceso de mano de obra a sectores más productivos forja desigualdad por la presión a la baja que el desempleo genera en los salarios, pero posteriormente, cuando culminen las posibilidades de absorción de trabajadores, se creará una presión al alza de salarios, provocando la caída en la desigualdad.

A partir de esta formulación, el economista ruso Simón Kuznets un año después, elabora el ejercicio estadístico de evaluar países de diferentes niveles de ingreso percápita vs variables de desigualdad. Encontró que la gran mayoría de países 
1 Ver FRIEDMAN, Milton. (1962). Capitalism and Freedom. The University of Chicago Press, Chicago ricos y de países muy pobres tendían a ser equitativos, mientras que países de ingreso medio mostraban niveles de desigualdad bastante altos. Este hallazgo empírico, fue representado posteriormente en una gráfica que identificaba en el eje de las ordenadas a la desigualdad y en el eje de las abscisas el ingreso percápita, lo cual arrojaba una curva en forma de U invertida.

Kuznets (1955) sugirió, que su resultado empírico tendría su explicación en que los países más pobres, dependían especialmente de la agricultura, una actividad con baja productividad pero mínima desigualdad. A partir de los procesos de migración de trabajadores rurales hacia las ciudades, el sector más productivo, el industrial, empezó a recibir trabajadores no calificados y con bajos salarios, lo cual promovía en esta etapa de transición alta inequidad (profundizada porque muchas personas del sector rural pasaban a conformar los cinturones de miseria de las ciudades) pero acompañada de un incremento importante de la producción (arrojando una relación positiva entre las dos variables). En la siguiente etapa, hay una gran cantidad de trabajadores que se ubican en el sector de mayores ingresos percápita (industrial), y una reducción del número de trabajadores en el sector agrícola, permitiendo un aumento de sus salarios relativos. En esta etapa la relación entre crecimiento y desigualdad se transforma a negativa. Este argumento se constituyó en lo que hoy se conoce como la Hipótesis de U invertida de Kuznets y ha servido de estrategia para la teoría neoclásica y monetarista en su defensa del capitalismo, cuando concluyen que solo este sistema generaría una alta movilidad social, garantizando la reducción de la desigualdad en su etapa más avanzada. ${ }^{1}$ La figura 1 ilustra la hipótesis.

Figura 1. Hipótesis de Kuznets

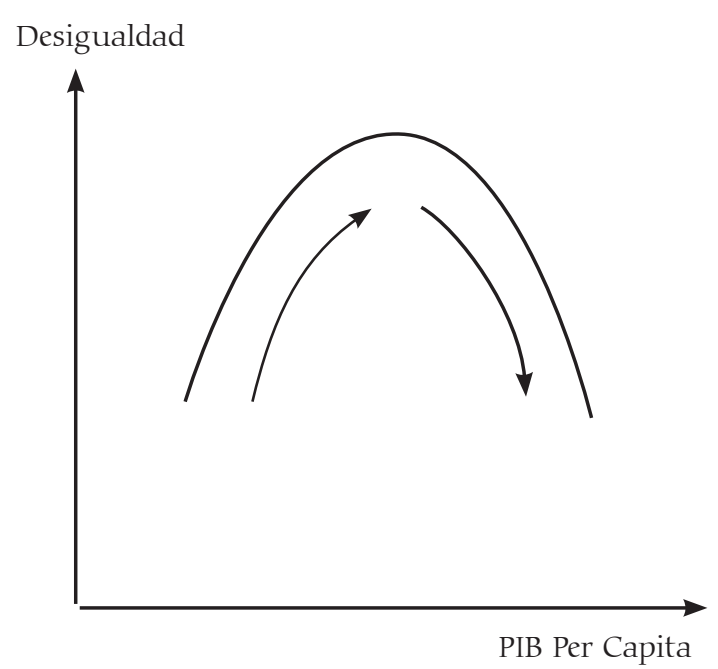


El problema implícito en los hallazgos y la hipótesis de Kuznets consiste en que emplea datos de corte transversal para explicar hechos dinámicos, por tanto sus conclusiones solo permiten establecer que hay una relación negativa entre desigualdad y crecimiento en un grupo de países pobres y una relación positiva en un grupo de países ricos. Pero no es posible hacer inferencia sobre la dirección de la causalidad. Es decir, no hay elementos para indicar qué ocurre primero o qué causa qué.

Casi simultáneamente a los hallazgos de Kuznets, surge la teoría de crecimiento de Nicholas Kaldor (1955) que contribuye a la explicación de la relación positiva de la curva. Explica que el crecimiento económico capitalista exige desigualdad en la distribución de la renta, ya que es necesario que aquellos que tienen una mayor propensión a ahorrar (los ricos -indicó-) concentren cada vez más ingresos para generar grandes cantidades de ahorro. Por ello, insiste Kaldor, en el proceso acumulativo capitalista debe existir algún tipo de compensación que reduzca la desigualdad generada por su propia dinámica. Sin embargo, se ha encontrado evidencia de que en países en desarrollo, especialmente de Latinoamérica, coinciden altos niveles de desigualdad con bajos niveles de ahorro como proporción del PIB, debido a que la clase alta realmente no posee una alta propensión a ahorrar, sino que destina gran parte de sus ingresos en bienes suntuosos (en su intento de emular, tal vez, a los vecinos del Norte) y/o en inversiones en el extranjero.

El discurso sobre las relaciones entre desigualdad y crecimiento se concentra a partir de los años 80, en la relación negativa que muestra la curva de Kuznets. Desde la teoría de crecimiento endógeno, se destaca a la acumulación de capital humano (y no sólo de capital físico) como el motor del crecimiento de largo plazo, debido a las externalidades positivas que éste produce en el uso y desarrollo de tecnología. Por tanto, mayor cantidad de personas educadas permite mayores niveles de crecimiento, lo cual implicaría una relación positiva entre crecimiento e igualdad. En este mismo sentido, es válido ampliar el concepto de capital humano a la idea de capacidades humanas de Amartya Sen. La expansión de dichas capacidades permite promover la libertad real de los individuos y a su vez permite generar mayor producción, la cual provee de nuevos ingresos para expandir cada vez más las capacidades humanas. ${ }^{2}$

La relación negativa se empezó a explorar, entonces como la posibilidad de que sea la desigualdad la que contrae el crecimiento y no como la caída de la primera a causa del aumento de la segunda, tal como lo indicaba Kuznets.

Desde la Economía Política, Albert Alesina y Dani Rodrick (1994), plantean que en un ambiente de desigualdad, donde la mediana de la población se encuentra por

2 SEN, Amartya y NUSSBAUM, Martha. (1993). La Calidad de Vida. (compiladores). Fondo de Cultura Económica. México. 
3 CENTRO DE INVESTIGACION Y DESARROLLO. (2007) Macroeconomía y Bienestar 2002-2006. Universidad Nacional de Colombia. Bogotá.

4 Existe una amplia literatura sobre la relación de causalidad positiva que se produce entre desigualdad y conflicto armado colombiano. Hay evidencia de que los departamentos y municipios más desiguales presentan mayores índices de violencia. Ver por ejemplo a SARMIENTO, A. Violencia y Equidad. (1999) En Revista Planeación y Desarrollo, Vol. XXX, y Rey, C. (2006). "Determinantes del Secuestro Extorsivo: Un enfoque Económico". Tesis de Grado de Maestría. Universidad Nacional de Colombia. debajo del ingreso promedio de los ciudadanos, en el momento de las elecciones, la población votará mayoritariamente por opciones que impliquen impuestos progresivos, es decir que graven más que proporcionalmente al que más tiene. Este hecho genera un desincentivo en la población de mayores ingresos y tiende a reducir su producción. En el mismo sentido señalan que, si la mayoría (el votante mediano) obtiene la mayor parte de sus ingresos del trabajo y no del capital, entonces habrá una preferencia por impuestos progresivos y por tanto, las clases altas reducen la producción. Como conclusión una alta desigualdad limita el crecimiento.

Esta propuesta tiene algunas dificultades. En primer lugar supone que la democracia funciona, que el resultado electoral corresponde a la preferencia real de las mayorías, que quienes aprueban el presupuesto (congresistas) no tienen intereses particulares que se puedan ver afectados por impuestos progresivos y que todos los ciudadanos votan. En segundo lugar, no tiene en cuenta el hecho de que los impuestos progresivos implican a su vez transferencias redistributivas que recibe la población en bienes como la educación, la cual incrementa el crecimiento de largo plazo, tal como ya se mencionó.

Desde la línea neokeynesiana también se postula una relación negativa entre desigualdad y crecimiento. Varios autores (Aghion 2001, Galor y Zeira 1993), han verificado que los problemas de información asimétrica que caracteriza los mercados crediticios, los convierte en mercados incompletos, ya que la aprobación de créditos depende especialmente de los ingresos del solicitante, lo que implica que la gran mayoría de la población no acceda a recursos para invertir en capital físico y/o en capital humano, a pesar de que dicha inversión podría haber garantizado el pago de la deuda. Esto limita el incremento de la demanda agregada y las potencialidades tecnológicas que podría tener el recurso humano que se dejaría de educar. Conclusión: las desigualdades permiten que solo accedan al crédito las clases altas (y que en algunos casos no lo necesitan), impidiendo el incremento de la producción que pudiese haber generado las demás clases. En Colombia este hecho es bastante palpable. El 20\% más rico de la población posee el 67\% del ahorro y acceden al 88\% del crédito por tarjeta. Mientras que el 20\% más pobre aporta el 2,3\% del ahorro total del país y accede al 0.43\% del crédito que se hace a través de tarjeta. ${ }^{3}$

El conflicto de clases también se convirtió en una guía para evaluar la causalidad en estudio. Robert Barro (1999) y Alesina (1996) formulan que ante desigualdades muy altas, una numerosa cantidad de individuos tiene incentivos a desarrollar actividades predatorias en vez de hacerlo en actividades productivas, ya que por un lado, los incentivos económicos de las primeras pueden superar al de 
las segundas y por otra parte, las grandes distancias de ingreso entre ricos y pobres produce un efecto envidia, dentro de la población menos favorecida, lo cual redunda en conflicto al buscar una redistribución forzada, que a su vez tiende a eliminar derechos de propiedad y los incentivos de los ricos a invertir por la pérdida de certidumbre. ${ }^{4}$ Esto se agrava si el conflicto implica un gasto ineficiente para la sociedad, tanto en el desarrollo de actividades violentas como en actividades defensivas. Conclusión: alta desigualdad genera inestabilidad y conflicto, lo cual retrasa el crecimiento.

Los cambios estructurales de liberalización financiera y comercial que han ocurrido entre la década de los 80 y 90, fueron promovidos en el Consenso de Washington como la pócima requerida para lograr el desarrollo. Se requería un sacrificio previo para consolidar el capitalismo, para que luego todas las clases recibieran las mieles de la riqueza generada por la libertad de los mercados nacionales e internacionales. Sin embargo las mieles no llegan. La relación positiva entre desigualdad y crecimiento volvió a aparecer y la U invertida se fue transformando en una N. Diversos estudios en Latinoamérica han demostrado que las nuevas condiciones financieras y comerciales han venido acompañadas de mayor desigualdad. Albert Berry (1997), sustenta que las empresas con uso intensivo de capital y en mano de obra calificada son las que han logrado traspasar las fronteras y han desplazado las demás actividades, especialmente la agricultura. La importación de maquinaria que sustituye mano de obra no calificada y la preferencia por mano de obra calificada ocasiona desempleo, presión a la baja en los salarios y poca posibilidad de negociación sindical.

Estos vaivenes entre relaciones positivas y negativas llevaron a Francois Bourguignon $(2005)^{5}$ a considerar que no es correcto hablar de tendencias distributivas vinculadas a mayor o menor crecimiento, se deben evaluar episodios o historias de desigualdad. Justamente esta conclusión se puede fortalecer con los resultados de la tabla 1. Ésta resume los hallazgos empíricos internacionales más importantes generados desde la formulación de la hipótesis de Kuznets hasta hoy. Se observa multiplicidad de conclusiones dependiendo de la metodología empleada (series temporales, corte transversal, datos panel), del período (desde el siglo XIX hasta finales de los 90) y los países evaluados (todos, solo pobres, solo subdesarrollados) y de las variables incluidas (depende del enfoque teórico del autor).

\footnotetext{
BOURGUIGNON, F., FERREIRA F.H. Y LUSTIG N. (Comp.). (2005). The microeconomics of income distribution dynamics in East Asia and Latin America. Washington, D.C: World Bank.
} 
Tabla 1. Resumen de Evidencia Empírica Internacional

\begin{tabular}{|l|c|l|}
\hline Autores & Año de Elaboración & Relación encontrada \\
\hline Weisskoff & 1970 & Indeterminada \\
\hline Robinson & 1976 & U invertida \\
\hline Knight & 1976 & U invertida \\
\hline Ahluwalia & 1976 & U invertida \\
\hline Chenery & 1979 & U invertida \\
\hline Williamson & 1980 y 1985 & U invertida \\
\hline Ram & 1989 & U invertida \\
\hline Anand y Kanbur & 1993 & Indeterminada \\
\hline Alesina y Rodrik & 1994 & Relación negativa \\
\hline Alesina y Perotti & 1996 & Relación negativa \\
\hline Deininger y Squire & 1998 & Relación negativa en los pobres \\
\hline Li y Zou & 1998 & Relación positiva \\
\hline Panizza & 1999 & Relación negativa \\
\hline Milanovic & 1999 & U invertida \\
\hline Barro & 1999 y 2000 & Relación negativa y U invertida en pobres \\
\hline Banerjee y Duflo & 2000 & Relación negativa \\
\hline Forbes & 2000 & Relación positiva \\
\hline Kanbur & 2000 & Indeterminada \\
\hline Cornia y Kïski & 2001 & N \\
\hline
\end{tabular}

Fuente: Elaboración del autor con base en Aghion (2001) y Panizza (1999)

\section{Hallazgos Colombianos}

El primer estudio elaborado para el caso colombiano fue realizado por Londoño (1995) a partir de una importante muestra estadística de más de 60 años. En su trabajo se acoge a los planteamientos de Kuznets, pero resalta la importancia del capital humano en el transcurrir de la curva. Indica que la desigualdad aumentó durante el proceso de transformación ocurrido a partir de la década de los 30

6 LONDOÑO, J.L. Y M. SZÉKELY. (1997). "Sorpresas distributivas después de una década de reformas", Documento de trabajo, Banco Interamericano de Desarrollo (BID), Washington DC.

7 Este documento hace parte de la compilación de casos de Bourguignon, F.; F.H. Ferreira y N. Lustig (eds.) (2005). The microeconomics of income distribution dynamics in East Asia and Latin America, World B a k , Washington, D.C. para llegar a su tope máximo a comienzos de los 60, momento a partir del cual empieza a descender rápidamente, gracias al rápido crecimiento económico, a la migración del sector con baja productividad hacia el sector industrial que equilibró los ingresos entre campesinos y obreros industriales y a los importantes avances en educación. Sin embargo, al extender su trabajo ${ }^{6}$ a la década de los 90, encuentra un nuevo incremento en la inequidad y concluye que las razones no obedecen a la liberalización comercial y financiera que arranca en los 80, sino a la caída de los progresos educativos y a la falta de transformación de la agricultura. Conclusión similar se encuentra en Carlos Vélez y otros (2005), ${ }^{7}$ y agregan que la alta fecundidad en los adolescentes y la inequidad en el acceso de las mujeres al mercado laboral profundizaron las desigualdades de los 90.

Sin embargo, han sido numerosos los trabajos que demostraron a finales de los 90 que las reformas aperturistas de los últimos 20 años, han generado incrementos en la desigualdad y aumentos en la pobreza rural en casi toda Latinoamérica. Dentro de los estudios se destacan Berry (1997), José A. Ocampo (1997) y Jorge I. González 
(2006) que coinciden en que la liberalización permitió la entrada de capital que reemplazó mano de obra, que la demanda de trabajo solo ocurrió por mano de obra calificada y que los trabajadores rurales perdieron al entrar a competir en fuerte desventaja con las técnicas y prácticas agrícolas de otros países.

Igualmente se encuentran estudios ${ }^{8}$ específicos de la pobreza rural, en los cuales se señala que la histórica concentración de la tierra en latifundios genera la baja productividad del campo y a su vez los bajos salarios rurales, lo cual promueve la emigración hacia la ciudad. Esta situación se agrava con la apertura que facilitó la importación de cultivos, empobreciendo cada vez más al campo.

Al respecto Miguel Székely y otros (2001) no encontraron evidencia estadísticamente significativa, entre la liberalización comercial sobre la desigualdad y la pobreza en Latinoamérica, pero detectaron evidencia de que la liberalización financiera tuvo efectos notables sobre el incremento de la desigualdad y la pobreza. En el mismo sentido Ocampo y Sánchez (2004) indican que la liberalización comercial permitió el aumento de las exportaciones, promoviendo así el crecimiento económico, pero no hallaron relación alguna entre esta liberalización y la desigualdad.

Finalmente vale la pena, destacar el trabajo de González y otros (2004), en el cual se evalúa la tendencia del crecimiento económico a reducir o no la pobreza en diferentes etapas. Muestran que en la mayoría de los años de estudios (1996 a 2004) el crecimiento no fue Pro Pobres, lo cual implica aumentos de la brecha entre quienes se beneficiaron del crecimiento y los pobres. Por ello insisten en que la forma de conseguir un crecimiento sostenible es emplear políticas redistributivas orientadas a elevar la consecución de tierra, capital, crédito y educación para los pobres, así como el diseño de un sistema tributario más progresivo.

\section{Evaluación de las Cifras}

Colombia es uno de los países más desiguales del mundo. De acuerdo con cifras de PNUD, ${ }^{9}$ el país ocupa el octavo puesto en el mundo y el tercero en América con un Gini de 0,57. En la actualidad es superado solo por Namibia, Lesoto, Bostwana, Sierra Leona, República Centroafricana, Haití y Bolivia. Esto implica que en el país, el decil más bajo solo accede al 0.07\% de los ingresos, mientras que el decil más alto recibe el 46.9\%.

La inequidad en la distribución de la tierra es más grave. La concentración reporta un Gini de 0,87. ${ }^{10}$ El 0,06\% de los propietarios rurales es dueño del 53,5\% de la tierra, lo que les permite poseer en promedio 18 mil hectáreas por persona. De forma contraria el $55.2 \%$ de los propietarios son dueños de solo el 1,7\% de la

\footnotetext{
Ver Puyana, A. (2002). "Riqueza petrolera, políticas macroeconómicas y la pobreza rural en Colombia". En: Pobreza, desigualdad social y ciudadanía. Compilado por Alicia Ziccardi. CLASCO. Buenos Aires (2003), y Gómez, Alcides. "Colombia: el contexto de la desigualdad y la pobreza rural en los noventa". En Cuadernos de Economía No 38. Universidad Nacional de Colombia. Bogotá.

9 PNUD. Human Develoment Reports $2007 / 2008$. Consultado en Página Web hdr.undp.org/ en/ Agosto de 2008

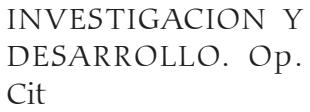

Palobrano. 9 Agosto/2008 
tierra, para un promedio de 3 hectáreas por propietario. Este hecho se refleja en la baja productividad de la tierra, ya que los minifundios terminan siendo sobreexplotados especialmente con productos de pancoger y los latifundios se subutilizan por medio de extensas zonas empleadas para ganado que emplean poca mano de obra.

De acuerdo con Londoño (1995), la desigualdad en los 60 había llegado al tope máximo de la U invertida de Kuznets, a partir de ese momento, el Gini empieza una caída vertiginosa hasta comienzos de los 80, cuando estalla la crisis financiera de 1982. En esta década el quintil más pobre recibía el 4,7\% de los ingresos y el quintil más rico accedía al 54,2\%, esto quiere decir que el 20\% de la población más rica obtenía 11,5 veces el ingreso del 20\% más pobre. La explicación a esta reducción ha sido los progresos educativos de los 60 y la amplia vinculación de la mano de obra no calificada al sector industrial, lo que permitió reducir la brecha por niveles de escolaridad.

En los 90 se desarrolla el proceso aperturista iniciado a finales de la década anterior promovido como la oportunidad de los colombianos para acceder al desarrollo. Sin embargo, la situación de inequidad empieza a revertirse. En esta década el decil más pobre recibía el 3,8\% de los ingresos y el más rico accedía al 58,1\%, de tal forma que el decil alto lograba 15 veces el ingreso del más bajo.

Gráfico 1 Evolución de la desigualdad

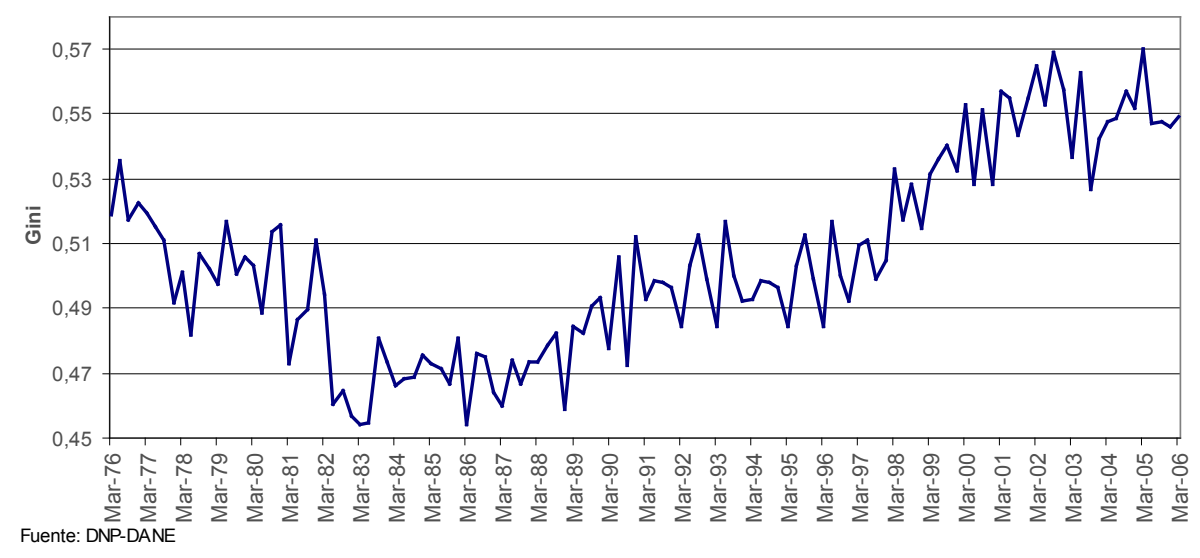

La economía cae a finales de los años 90 en la crisis más grave de los últimos 70 años y con ello el coeficiente de Gini toma un ascenso acelerado que lo sitúa en adelante siempre por encima de 0,53. Luego, el ciclo económico inicia nuevamente su ascenso, pero la desigualdad no cedió, por el contrario, registró los picos de 0,57 en el 2002 y en el 2005, los niveles de desigualdad más altos registrados desde los años 60. En la primera década del siglo XXI el decil más pobre recibe 
solo el 3\% de los ingresos y el más rico obtiene el 62\%, lo que quiere decir, que el 10\% más rico gana 20 veces lo que gana el 10\% más pobre (casi el doble de lo ocurrido en los 80).

Colombia es un país que tiende a la desigualdad y que a pesar del crecimiento, no muestra flexibilidad al cambio. Mientras casi todos los países de Latinoamérica lograron aprovechar el auge con mejoras en la distribución, solo hubo tres países que generaron crecimiento con inequidad, República Dominicana, México y Colombia. ${ }^{11}$ Es decir la pendiente negativa de la U invertida de Kuznets no llegó.

Esta afirmación sugiere que la recompensa por el sacrificio exigido en el ascenso de la curva nunca apareció. Al revisar la gráfica 2 que relaciona desigualdad y PIB Percápita en los últimos 25 años, se confirma lo dicho. Se observa que luego de la caída en el PIB del 82, el país nuevamente empezaba su ascenso en la U invertida sobre finales de los 80, es decir los colombianos, al igual que en los 50 debían volver a sacrificar la equidad en aras de mayor crecimiento. Luego se llega a un tope máximo en 1993 y parece que el sacrificio había terminado, se empieza a caer sobre la parte negativa de la curva hasta 1997, año en el cual se consigue combinar reducciones de la desigualdad junto con aumentos de la actividad económica. Se genera U invertida completa.

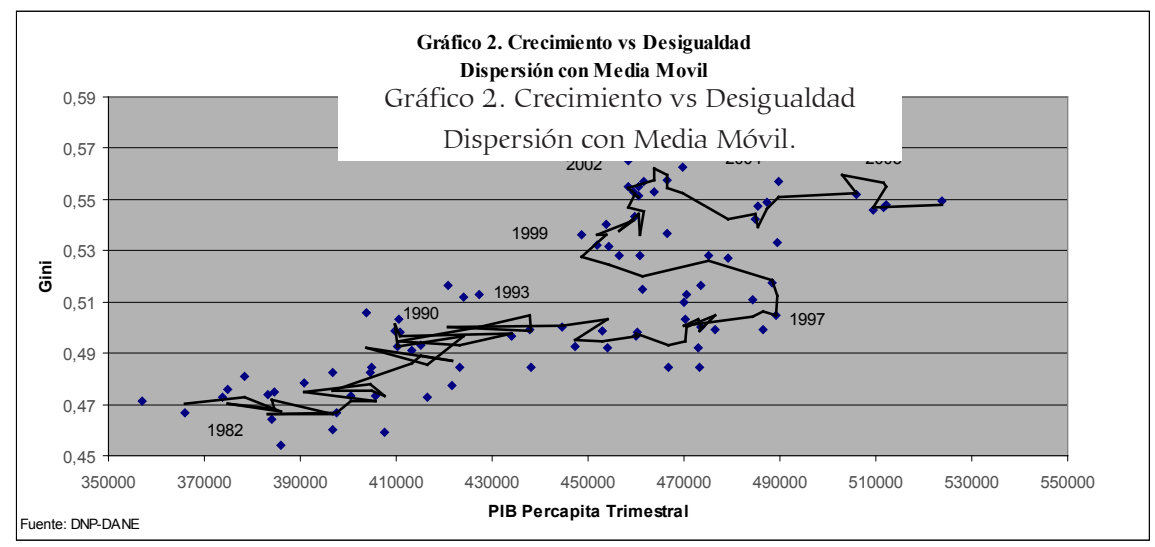

Sin embargo, a partir de 1998 empieza un retroceso. Nos devolvemos sobre la parte negativa de la U invertida, pero lo hacemos de abajo hacia arriba y con una pendiente mayor, es decir, se reduce el PIB Percápita y se eleva la desigualdad a un nivel mucho mayor que el de 1993. Posterior a la salida de la crisis, el país no vuelve a bajar sobre la U invertida, sino que se mantiene la desigualdad, a pesar del mayor crecimiento. El país se recuperó, pero sin resolver el problema distributivo. La reducción de la inequidad se aplaza una vez más, vamos de sacrificio tras sacrificio a la espera de que alguna vez nos llegue el efecto derrame de la riqueza.

11 CEPAL. Panorama Social de América Latina 2007. (2007). CEPAL. Consultado en Pagina Web www. eclac.org 


\section{Producción, Empleo e Ingresos}

El determinante clave del crecimiento en Colombia fue la situación internacional favorable, ya que coincidió un aumento de la demanda externa que incrementó nuestras exportaciones y la entrada de remesas, el aumento de precios en bienes claves como el café, ferroníquel, petróleo y carbón, que permitieron un incremento en el valor de las exportaciones y reducción de la tasa de interés internacional que facilitó la inversión mundial. Los hechos anteriores sumados al aumento del gasto público y el aumento del consumo vía crédito permitieron un incremento de la demanda agregada interna a un ritmo mayor que la producción interna,

${ }^{12}$ CID. Op. cit

${ }^{13}$ Cálculos a partir de Cuentas Nacionales del DANE.

${ }^{14}$ Del total de empleos generados en toda la economía entre 2001 y 2005 , solo el $1,21 \%$ fue creado por los establecimientos financieros. (Cálculos del autor con base en DANE)

15 El 70\% del empleo en construcción es informal, en el caso del comercio, hoteles y restaurantes la informalidad es de $77 \%$ y en transporte y comunicaciones es de 62\%. (Cálculos del autor con base en DANE)

${ }^{16}$ El nivel de subempleo mas alto se encuentra en actividades como la construcción, el transporte, comunicaciones, comercio, hoteles y restaurantes, donde del total de ocupados el $42 \%$ en promedio se encuentra en condiciones de subempleo. (Cálculos del autor con base en DANE) lo cual implica una presión al alza sobre los precios nacionales y/o comprar en el extranjero el exceso, es decir importar. ${ }^{12}$

Por otra parte, se presenta una tendencia a terciarización (movilidad hacia los servicios y el comercio) de la economía. Además de la construcción, los sectores con mayor crecimiento en la etapa de recuperación son el comercio, restaurantes y hoteles, transporte, comunicaciones y servicios financieros, mientras que los que menos han crecido corresponden al sector primario y secundario de la economía (agricultura, pesca, minería e industria). El sector primario solo aporta el 12.9\% de la producción, el sector secundario el 20\%, mientras que el 67\% restante es generado por servicios y comercio. El énfasis en esta terciarización ha sido puesto en el sector financiero, que con una participación del $17.2 \%$ se consolida como la actividad más importante dentro de la producción nacional. ${ }^{13}$

El desempleo reciente no ha variado de acuerdo con el ciclo económico. Mientras que a comienzos de los 80 y mediados de los 90 se combinaba alto crecimiento con desempleo de un dígito, en el 2007 se registró el mejor nivel de crecimiento de los últimos 30 años, pero el desempleo sigue siendo mayor que lo ocurrido en plena crisis de 1982. De hecho el desempleo actual solo es inferior a lo reportado entre 1998 y 2000, pero hay que resaltar que a partir de este momento ocurrió un cambio de metodología en el DANE que bajó automáticamente la tasa.

El desempleo no ha bajado, entre otros motivos, porque los sectores que han jalonado el crecimiento económico no generan empleo ${ }^{14}$ o generan fundamentalmente empleo informal ${ }^{15} \mathrm{y}$ subempleo. ${ }^{16}$ Por otra parte, el aumento de las importaciones y la facilidad que el gobierno le ha dado a la inversión extranjera y el libre comercio, podría estar provocando desempleo porque, o bien se sustituye la producción nacional por producción extranjera, o bien se importa bienes de capital que desplazan mano de obra no calificada.

De acuerdo con cifras del DANE, el poder adquisitivo de los trabajadores se ha 
reducido. En la gráfica 3 se observa que los ingresos reales de los trabajadores aumentaron a mediados de los 90, momento en el cual la desigualdad tiende a bajar, mientras que a partir del 2000 los ingresos reales (independientemente del nivel de formación) empiezan a caer, coincidiendo con el nuevo repunte vertiginoso de la desigualdad, a tal punto que los niveles de poder adquisitivo actuales son inferiores a los que tenían los trabajadores a comienzos de los 80 .
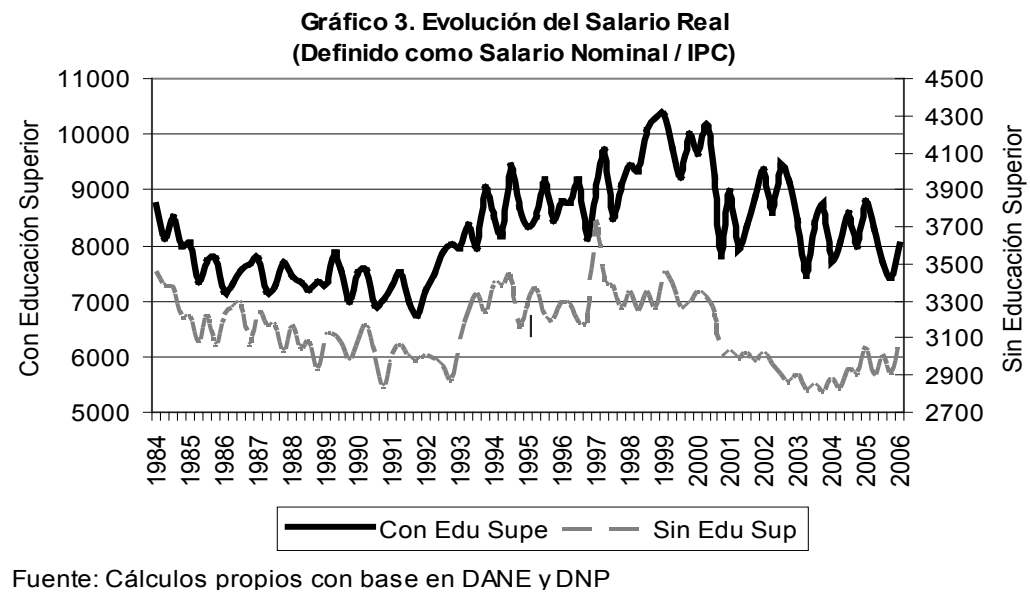

Por otro lado, es importante resaltar que a partir del 2001 la proporción de los trabajadores que ganan menos de un salario mínimo, crece de forma considerable, ubicándose en $32 \%$ para el total de trabajadores y $41 \%{ }^{17}$ para los que carecen de educación superior. De acuerdo con el BID, ${ }^{18}$ Colombia es el segundo país (después de Nicaragua) de Latinoamérica donde más se evade el salario mínimo. En la zona rural se evade en un 54\% y en la zona urbana $17 \%$.

Desde la salida de la crisis hasta hoy, el ingreso real de los trabajadores de la industria ha caído en 8\%, el de la construcción en 19\% y el del comercio se reduce en $8 \%$, mientras que el de los servicios financieros y comunales creció en 19\% y el transporte solo creció en un 2\%. De tal forma que el 54\% de los trabajadores urbanos perdieron capacidad adquisitiva, el 10\% quedó igual y el 36\% aumentó. ${ }^{19}$ Esto exige una nueva adaptación de la Hipótesis de Kuznets, ya que la transformación no ocurre desde el agro hacia la industria, sino que ahora se mueve desde la segunda hacia los servicios, producto de la tendencia a la terciarización de la economía nacional.

De otra parte, entre 1997 y 2005 la productividad laboral en la industria creció en un $39 \%$, el PIB industrial en un $28 \%$ pero el salario real solo lo hizo en un $8 \% .{ }^{20}$ En este sentido, no hay consistencia con la teoría neoclásica, ni con los "acuerdos" salariales sobre el mínimo, que señalan la existencia de una correlación fuerte entre salario real y productividad. Este hecho implica que los propietarios

17 Cifras obtenidas de LASSO, F. y LÓPEZ, H. (2008). Salario mínimo, salario medio y empleo asalariado privado en Colombia. Borradores de Economía No 484. Banco de la República. Bogotá.

${ }^{18}$ Ibid

${ }^{19}$ Cálculos del autor a partir de DNP-DANEEncuesta Continua de Hogares.

${ }^{20}$ Cálculos del autor a partir de la Encuesta Anual Manufacturera efectuada por el DANE. 
de las empresas se quedan con cada vez más parte del producto, lo que amplía nuevamente la brecha entre capitalistas y trabajadores.

Se puede fortalecer esta hipótesis, revisando los ingresos del capitalista en el proceso productivo vs remuneración de los trabajadores. El ingreso del capitalista se encuentra representado en las Cuentas Nacionales a través del Excedente Bruto de Explotación (EBE) y el salario de los trabajadores a través de la Remuneración de los trabajadores (RET). En las gráfica 4 y 5 se expresa la relación EBE/RET y su evolución en los últimos 14 años. ${ }^{21}$ El aumento de esta relación, significa que el capitalista se apropia cada vez más de una mayor parte de la producción en comparación con lo que reciben sus trabajadores.

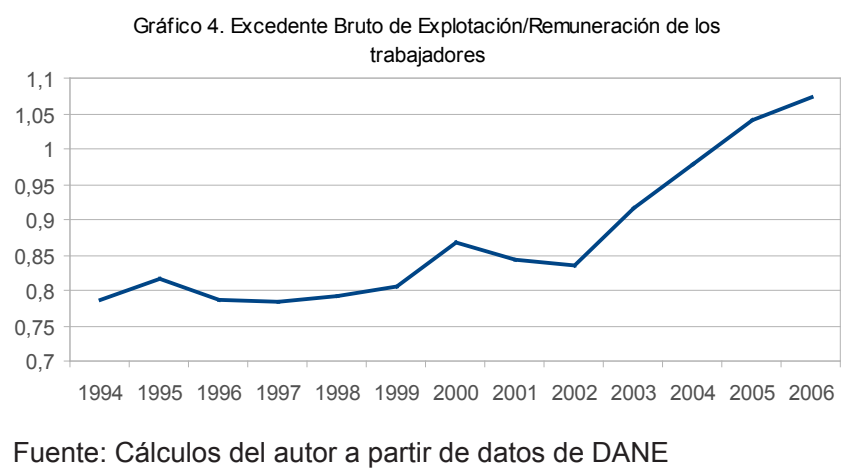

Obsérvese en la gráfica 4 que la evolución de este indicador es similar a lo ocurrido con el Gini. Un nivel ligeramente bajo en 1996 y 1997, años que coinciden con un crecimiento económico con tendencias a la equidad. Posterior a la crisis, la EBE/RET inicia un pequeño ascenso que luego se acelera notablemente a partir del 2003. Nuevamente se evidencia lo altamente inequitativo del crecimiento reciente. Los capitalistas reciben una proporción cada vez más grande de la renta, en contra vía de lo que ocurre con el trabajador que participa cada vez menos de los resultados de la producción.

Los sectores con una EBE/RET más baja son el agrícola y el comercio, mientras que la industria, la construcción y la minería son las de mayor registro, lo cual

21 Se debe tener cuidado con la interpretación del valor porque depende de la intensidad de uso del capital y de la cantidad de empleados. se explica por las diferencias en la intensidad de uso del capital. Para el periodo de crecimiento con tendencias a la equidad (1997) la participación de los trabajadores mejoró en la industria y la minería. En 1999 hubo un repunte en los beneficios para el capitalista en el subsector del petróleo, pero a partir del 2003 inicia una evolución fuerte y sostenible de la EBE/RET en todos los sectores, destacándose el aumento en la participación del capitalista en la industria, la minería, y el comercio que duplicaron el indicador frente a 1994, mientras que el sector agrícola 
fue el único en reducir la relación de distribución de la renta con respecto a ese mismo año.

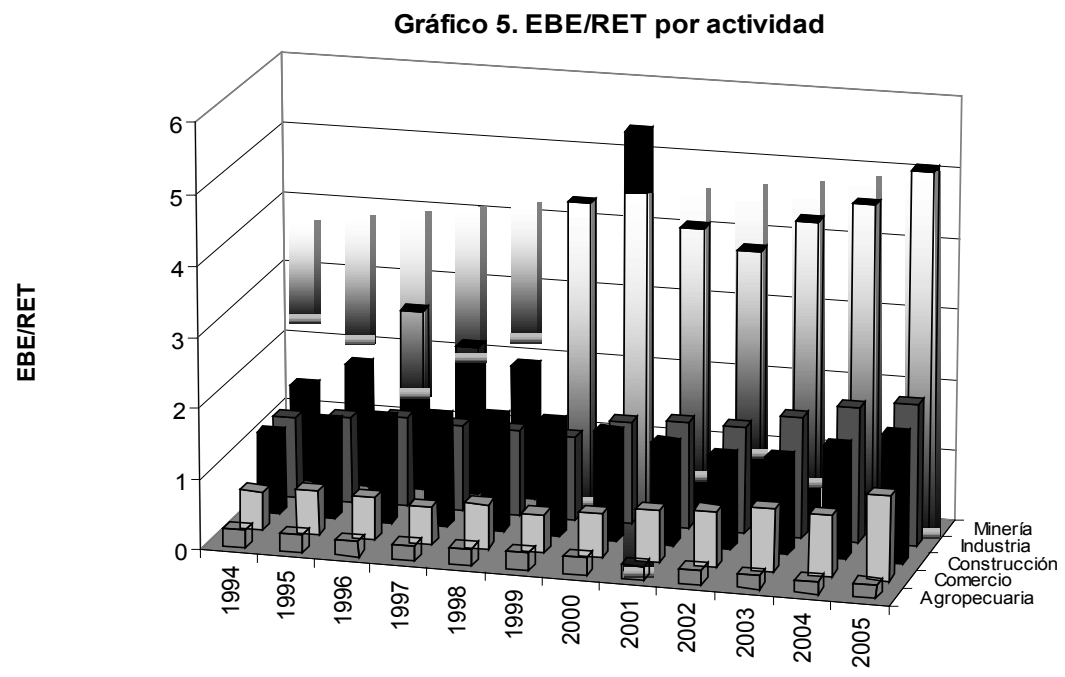

Fuente: Cálculos del autor a partidslapdatos de DANE

\section{Nuestras Estimaciones y Resultados}

Para verificar la hipótesis de Kuznets en Colombia y sus posibles variaciones, se formula un modelo econométrico que extiende la hipótesis de la U invertida, por medio de la inclusión de nuevas variables macroeconómicas que pueden tener o no alguna incidencia sobre la desigualdad, de acuerdo a los argumentos teóricos y elementos a priori. Este modelo permitirá tener evidencia sobre la existencia de relación entre las variables explicativas y la variable exógena, así como el grado y tipo de relación.

Fijando a la desigualdad como la variable dependiente se plantearon dos ejercicios de modelación por el método de mínimos cuadrados. Uno con una serie trimestral que va desde 1985 hasta 2006 y otro que recoge solo el tiempo de apertura (1990 en adelante). Se utilizaron las siguientes variables explicativas e hipótesis:

- PIB percápita (pesos de 1994). Se espera una relación positiva y se adhiere la variable PIB percápita al cuadrado para recoger el posterior efecto negativo de la U invertida. Además se incluyó la misma variable al cubo para verificar alguna posible relación posterior a la Hipótesis de Kuznets.

- Salarios reales de personas con baja escolaridad. Se espera una relación inversa.

- Tasa de Desempleo. Se espera relación directa.

- Inflación. Se espera relación directa 
- Profundización financiera (M2/PIB): Esta variable recoge de forma precaria la cantidad de crédito en manos del público como proporción de la renta nacional. Se espera relación inversa, porque sería una aproximación al supuesto de que hay mayor acceso al crédito.

- Importaciones de consumo (como proporción del PIB). La entrada de bienes extranjeros reduciría mercado a los bienes nacionales, generando desempleo, entonces se espera relación positiva.

- Importaciones de Bienes de capital (como proporción del PIB): La entrada de este tipo de bienes desplazaría mano de obra, entonces se espera relación positiva.

Este ejercicio arrojó como resultado:

\begin{tabular}{|c|c|c|c|c|}
\hline \multicolumn{5}{|c|}{ Tabla 2 } \\
\hline \multicolumn{2}{|c|}{ Resultados de econometría variable dependiente: GINI } \\
\hline Variable & \multicolumn{2}{|c|}{$1985-2006$} & \multicolumn{2}{c|}{$1990-2006$} \\
\hline PIB Percapita & Coeficiente & Prob. & Coeficiente & Prob. \\
\hline PIB Percapita ${ }^{2}$ & 0.0078 & 0.00 & 0.0092 & 0.00 \\
\hline PIB Percapita & $-1.5 \mathrm{E}-05$ & 0.00 & $-2.5 \mathrm{E}-05$ & 0.00 \\
\hline Salario Real de baja es colaridad & -0.1107 & 0.00 & -0.0769 & 0.00 \\
\hline Tasa de desempleo & 0.3624 & 0.00 & 0.3695 & 0.02 \\
\hline Tasa de Inflación & -0.1091 & 0.15 & -0.1052 & 0.27 \\
\hline mportación de Bienes de Consun & 0.5492 & 0.25 & 1,0896 & 0.04 \\
\hline Importación de Bienes de Capita & -0.1636 & 0.74 & -0.7257 & 0.18 \\
\hline Profundización Financiera & -0.0118 & 0.66 & -0.0017 & 0.95 \\
\hline R Cuadrado & & 0.83 & & 0,81 \\
\hline
\end{tabular}

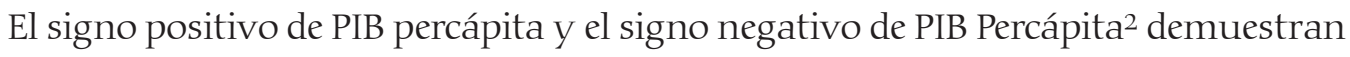
que hay evidencia del cumplimiento de la Hipótesis de Kuznets, sin embargo la historia no termina allí. El signo positivo de PIB percápita ${ }^{3}$ implica que luego de la caída de la desigualdad, empieza una extensión de la curva, pero nuevamente con pendiente positiva, es decir se combina una vez más crecimiento con mayor inequidad. La U invertida se transforma en $\mathrm{N}$ evidenciando que las transformaciones económicas recientes alteran el resultado de Kuznets, por tanto la modalidad de crecimiento que el país eligió exige una vez más sacrificar la equidad en aras de la eficiencia. La figura 2 ilustra el resultado. 
Figura 2

Desigualdad

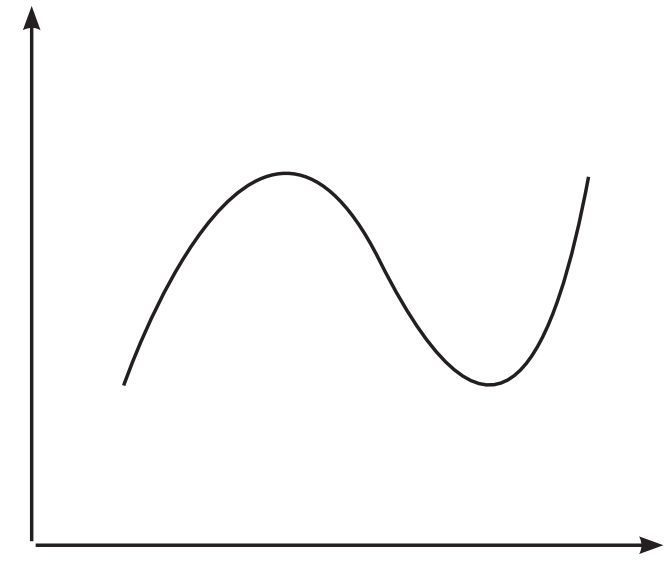

PIB Percápita

Con respecto a la influencia del mercado laboral, se comprueba, que aumentos del salario real de la población con menor escolaridad, disminuye la desigualdad, e incrementos en la tasa de desempleo la empeora, tal como se había previsto en las relaciones hipotéticas planteadas. En los dos casos se logra significancia estadística. Esto es un indicativo de que el tipo de crecimiento colombiano se basa en sectores poco generadores de empleo y de bajos salarios, con lo cual promueve la desigualdad.

El incremento de los precios no mostró relación estadísticamente significativa con la desigualdad, de hecho, el signo que resulta contradice los tradicionales argumentos monetarios sobre los efectos desfavorables de la inflación sobre la desigualdad. Respecto a esto César Merchán (2002) ${ }^{22}$ considera que la política monetaria contractiva para el control de la inflación ha generado efectos regresivos sobre la desigualdad, porque el aumento implícito de las tasas de interés podría generar desempleo.

${ }^{22}$ Se encuentra un No se halló evidencia estadísticamente significativa del impacto de importación de bienes de consumo, ni de importación de bienes de capital. Sin embargo la regresión elaborada para el periodo de apertura, generó evidencia significativa para la importación de bienes de consumo y mantuvo la no significancia para los bienes de capital. Esto indica que desde los 90 la entrada de productos extranjeros podría estar desplazando productos nacionales que generan empleo y mayores salarios para los trabajadores colombianos. Por el contrario, sorprende el signo negativo (aunque no significativo estadísticamente) de los bienes de capital, porque indicaría que la entrada de este tipo de bienes reduce la inequidad, sugiriendo que resultado similar en MERCHAN, Cesar. (2002). "Incidencia de la inflación sobre la distribución del ingreso. El caso colombiano". En: Cuadernos de Economía No 37. Universidad $\mathrm{Nac}$ ional de Colombia. Bogotá. 
esta importación permite la creación de nuevos empleos y/o mejores salarios.

La profundización financiera arrojó el signo esperado, pero la relación resultante fue estadísticamente no significativa.

\section{Dirección de la Causalidad}

Por medio del test de causalidad de Granger (cuatro rezagos) se encontró evidencia de que la desigualdad incide sobre el crecimiento y no al revés. Por ello, se planteó una nueva ecuación, en la cual se fija como variable endógena al PIB percápita y como variable explicativa el coeficiente de Gini. Además se agrega un Gini al cuadrado y otro al cubo, con el fin de verificar la relación no lineal entre las dos variables. El resultado fue el siguiente:

Tabla 3. Resultados con nueva causalidad
\begin{tabular}{|c|c|c|}
\hline \multicolumn{2}{|c|}{$1985-2006$} \\
\hline Variable Dependiente: PIB Percápita \\
\hline Variable & Coeficiente & Prob. \\
\hline GINI & -5055.8 & 0,002 \\
\hline GINI $^{2}$ & 23001.1 & 0,000 \\
\hline GINI $^{3}$ & -22232.0 & 0,000 \\
\hline R cuadrado & & 0,61 \\
\hline
\end{tabular}

Los datos muestran que la desigualdad actual contrae el crecimiento. Hay un instante en el cual, la inequidad (como lo sugiere Kaldor), facilita el crecimiento. Sin embargo, el signo de Gini ${ }^{3}$ evidencia que esta relación directa llega a un máximo, a partir del cual la desigualdad retarda el crecimiento o lo hace insostenible, entonces el aumento del PIB percápita colombiano reciente sería mayor, si se hubiese visto acompañado de algún tipo de política redistributiva. La figura 3 ilustra el resultado.

Figura 3

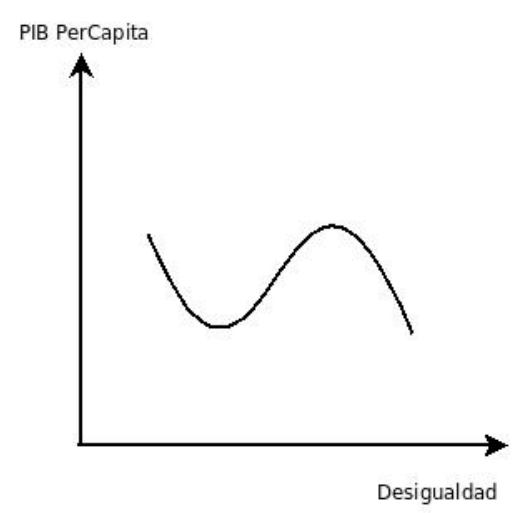




\section{Comentarios Finales}

De acuerdo con los resultados del modelo es posible afirmar que el aumento de la desigualdad en Colombia ocurrida durante los últimos 20 años obedece al incremento de una producción que no generó empleo y redujo los salarios reales de los trabajadores de baja escolaridad. Este hecho es un factor clave para que la U-Invertida de Kuznets retome nuevamente su ascenso hacia mayor crecimiento, pero con alta inequidad, tal como se demuestra en los hallazgos no-lineales de la regresión. Además, la historia se agravó a partir de los años 90, debido al efecto desfavorable que provocó la importación de bienes de consumo. Las demás variables incluidas en el modelo no presentaron relación estadísticamente significativa.

El planteamiento de Kuznets no es una ley. Cada país tiene su propia historia de desigualdad vs crecimiento. Se propone como hipótesis que la curva de U-Invertida de Kuznets no se cumple en Colombia por tres motivos: a) La transformación económica de los últimos 20 años extendió el sacrificio de la mayoría de los colombianos, gracias a la entrada de productos extranjeros en condiciones de competencia desventajosa para los nacionales, aumentando el desempleo y reduciendo los salarios reales de los colombianos, b) La industria nacional no se transformó, no logró absorber mano de obra de los sectores menos productivos y no mejoró los salarios relativos de los trabajadores, por el contrario la apertura de fronteras alimentó la lentitud de su crecimiento y c) La terciarización precaria de la economía está desplazando trabajadores a sectores con baja productividad, con empleo de baja calidad y de reducidos salarios.

Finalmente, existen diversas formas de crecer. Unas inequitativas y otras redistributivas. Elegir cual depende de la función social de bienestar de un país y/o de sus gobernantes. En términos de González (2006) la elección depende del grado de aversión a la inequidad de cada sociedad. La elegida por Colombia, demuestra una preferencia utilitarista con poca o nula aversión, en donde basta que alguno se enriquezca para garantizar crecimiento, así esto requiera empeorar la situación de otro. Por el contrario una función social de tipo Rawls, exige que el menos favorecido avance para que la sociedad esté en una mejor condición, así ello implique sacrificar algo de los ricos. Aunque esta elección implicaría anteponer elementos éticos a la economía ortodoxa, y a renunciar a varios supuestos del fundamentalismo de mercado, los datos de este documento invitan a pensar en la posibilidad de una elección redistributiva (por ejemplo de tipo rawlsiano) capaz de garantizar un crecimiento que además de justo, sea sostenible. 


\section{BIBLIOGRAFIA}

AGHION, Philippe y WILLIAMSON Jeffrey. (2001). Growth, inequality and globalization. Cambridge University Press. Cambridge.

ALESINA, A. Y RODRIK, D. (1994). "Distributive politics and economic growth". En Quarterly Journal of Economics, 109, núm. 2.

ALESINA, A., y otros. (1996). "Political instability and economic growth" en Journal of Economic Growth, núm. 1.

BARRO, R. J. (1999). "Inequality, growth, and investment", NBER Working Paper 7038.

BERRY, Albert. (1997). "The income distribution threat in Latin America". En Latin American Research Review, Vol. 32, No 2.

CORNIA, Giovanni. (2004). Inequality, growth and poverty in an era of liberalization and globalization. Oxford University Press. Oxford.

EKELUND, B. y HERBERT, R.F. (2006). Historia de la teoría económica y de su método. McGraw Hill. Madrid.

GALOR, O. y ZEIRA, J. (1993). "Income distribution and macroeconomics". En Review of Economic Studies, Vol 60.

GONZÁLEZ, Jorge. (2006). Ética, economía y políticas sociales. Corporación Región. Medellín.

KALDOR, N. (1955). "Alternative theories of distribution", Review of Economic Studies, v. 23.

KUZNETS, S. (1955). "Economic growth and income inequality", American Economic Review, Vol 65.

LEWIS, A. (1954). "Economic development with unlimited supplies of labour" Manchester School, Vol. 22, Manchester.

LONDOÑO, Jorge. (1995). Distribución del Ingreso y Desarrollo Económico. Colombia en el Siglo XX. Bogotá: FEDESARROLLO.

OCAMPO J. y otros. (1997). Macroeconomía, ajuste estructural y equidad en Colombia. 1978-1996. Santiago de Chile: CEPAL. 
OCAMPO J. y otros. (2004). Crecimiento de las exportaciones y sus efectos sobre el empleo, la desigualdad y la pobreza en Colombia. Documentos CEDE. Bogotá.

OCHOA, Lina. (2002). "Crecimiento y distribución: una evaluación de la hipótesis de Kuznets". En Cuadernos de Economía No 38. Universidad Nacional de Colombia. Bogotá.

PANIZZA, Ugo. (1999). Income inequality and economic growth: evidence from American data. Washington, D.C: BID.

SZEKELY, M y otros. (2001). Pobreza, desigualdad y liberalización comercial y financiera en América Latina. BID. Washington, D.C.

BIOGRAFIA

CAMILO REY SABOGAL 